COLO-HEP-98/424

hep-th/9905080

May 1999

\title{
Tachyon Condensation in Rotated Brane Configurations
}

\author{
S. P. de Alwis' \\ Department of Physics, Box 390, University of Colorado, Boulder, CO 80309.
}

\begin{abstract}
The decay of rotated brane configurations and the corresponding condensation of tachyons is discussed. In a certain IIB orbifold case a heuristic argument about the mass of the state living on the fixed plane is made. When the rotation angle is $\pi$ this mass agrees with that obtained by Sen.
\end{abstract}

\footnotetext{
${ }^{1}$ e-mail: dealwis@pizero.colorado.edu
} 


\section{Introduction}

In the last year there has been significant progress in understanding certain non-BPS states in string theory. In particular it has been shown by Sen [1]-[1] that there are unstable non-BPS Dp-branes with even (odd) p in type IIB (IIA) branes and a stable non-BPS D-particle in type I theory. In the case of IIA and IIB too one could stabilize the non-BPS branes by dividing out by certain $Z_{2}$ symmetries. A stable D-particle state in type IIB was explicitly constructed using the boundary state formalism in [5]. The relation of these developments to K-theory has been pointed out in [6] where some new insights have been obtained. These have been analyzed using the boundary state formalism in [7].

All the above considerations were made in the context of (anti-) parallel brane configurations. In this paper we wish to consider situations where branes are not parallel (or anti-parallel). We will begin with a review of the evaluation of the partition function for open strings with ends on two D-branes, one of which is rotated with respect to the other, and the observation that there is a tachyon in the system for general angles when the distance between the branes is less than a certain critical value. We argue that (at least when the brane directions are compact) this tachyon represents an instability of the system to decay into a BPS brane which is obtained from the vector sum of the charges of the original branes. Next we construct boundary states corresponding to such D-branes and verify that they are consistent with open string closed string duality. We consider, closely following Sen's work [2], this system in type $I I B / Z_{2}$ where the $Z_{2}$ is generated by a reflection in four coordinates times $(-1)^{F_{L}}$, compactify one direction (which is common to both branes) on a circle, and put a Wilson line on it. Upon tachyon condensation, it is argued that we now have a solitonic state on the orbifold fixed plane whose mass depends on the angle of rotation between the D-branes, in addition to a brane that stretches between the two fixed planes. When the angle is $\pi$ (corresponding to one of the branes being an anti-brane) the charge and hence mass of the extended brane is zero, and the mass of the soliton on the fixed plane is equal to that calculated by Sen. However unlike in this latter case, for a general angle we have not been able to establish these results rigorously, since it has not been possible to find a conformal field theory argument.

The calculations are performed in the double Wick rotated light cone gauge formalism adopted in [9], [2]. Strictly speaking since in this formalism the time direction is transverse to the brane (all directions parallel to the brane have positive metric) the configurations are really $(\mathrm{p}+1)$-instantons. However we will assume (as in [9], [2]) that this is equivalent to a calculation with p-branes when done with all the ghost complications. We will take the original time coordinate to be $x^{2}$ and the light cone directions after Wick rotation to be $x^{0}, x^{1}$.

\footnotetext{
${ }^{1}$ For recent reviews of these developments see [8].
} 


\section{The open string partition function for rotated D- branes}

We begin by quoting a calculation given in [10] (section 13.4) for the open string partition function, where strings have one end attached to a three-brane and the other to another three brane, rotated with respect to the first. Actually the treatment in 10 is for a four-brane, but in our framework this is to be interpreted as a four instanton that is equivalent to a double Wick rotated 3-brane. One of the three branes is oriented along the $x^{2}, x^{4}, x^{6}, x^{8}$ (it should be remembered that $x^{2}$ is really time) directions and the other is rotated with respect to this by an angle $\phi_{1}$ in the $x^{2}-x^{3}$ plane, $\phi_{2}$ in the $x^{4}-x^{5}$ plane etc with $0<\phi_{i}<\pi$. In our convention the first rotation would correspond to a Lorentz transformation when we Wick rotate back. The result of the calculation is

$$
\begin{aligned}
\int \frac{d t}{2 t} \operatorname{tr}_{N S} e^{-2 \pi t H_{O}} & =\int_{0}^{\infty} \frac{d t}{t} \frac{e^{-\frac{t y^{2}}{2 \pi \alpha^{\prime 2}}}}{\left(8 \pi^{2} \alpha^{\prime} t\right)^{\frac{1}{2}}} \prod_{i=1}^{4} \frac{\theta_{00}\left(\frac{i \phi_{i} t}{\pi}, i t\right)}{\theta_{11}\left(\frac{i \phi_{i} t}{\pi}, i t\right)} \\
\int \frac{d t}{2 t} \operatorname{tr}_{N S}(-1)^{F} e^{-2 \pi t H_{O}} & =\int_{0}^{\infty} \frac{d t}{t} \frac{e^{-\frac{t y^{2}}{2 \pi \alpha^{\prime 2}}}}{\left(8 \pi^{2} \alpha^{\prime} t\right)^{\frac{1}{2}}} \prod_{i=1}^{4} \frac{\theta_{01}\left(\frac{i \phi_{i} t}{\pi}, i t\right)}{\theta_{11}\left(\frac{i \phi_{i} t}{\pi}, i t\right)} \\
\int \frac{d t}{2 t} \operatorname{tr}_{R} e^{-2 \pi t H_{o}} & =\int_{0}^{\infty} \frac{d t}{t} \frac{e^{-\frac{t y^{2}}{2 \pi \alpha^{\prime 2}}}}{\left(8 \pi^{2} \alpha^{\prime} t\right)^{\frac{1}{2}}} \prod_{i=1}^{4} \frac{\theta_{10}\left(\frac{i \phi_{i} t}{\pi}, i t\right)}{\theta_{11}\left(\frac{i \phi_{i} t}{\pi}, i t\right)} \\
\int \frac{d t}{2 t} \operatorname{tr}_{R}(-1)^{F} e^{-2 \pi t H_{O}} & =\int_{0}^{\infty} \frac{d t}{t} \frac{e^{-\frac{t y^{2}}{2 \pi \alpha^{\prime 2}}}}{\left(8 \pi^{2} \alpha^{\prime} t\right)^{\frac{1}{2}}} \prod_{i=1}^{4} \frac{\theta_{11}\left(\frac{i \phi_{i} t}{\pi}, i t\right)}{\theta_{11}\left(\frac{i \phi_{i} t}{\pi}, i t\right)}
\end{aligned}
$$

where we have taken the distance between the branes to be $y$. Let us specialize to the case when three of the angles are zero. The $\theta_{11}$ function in the denominators blow up when $\phi=0$ and one needs to substitute $i \theta_{11}\left(\frac{i \phi t}{\pi}, i t\right) \rightarrow L \eta(i t)^{-3}\left(8 \pi^{2} \alpha^{\prime} t\right)^{-\frac{1}{2}}$ (equation (13.4.24) of [10]) for the three parallel directions (each of whose size is taken to be $L$ ). Then we get for the potential,

$$
V=L^{3} \int_{0}^{\infty} \frac{d t}{t}\left(8 \pi^{2} \alpha^{\prime} t\right)^{-2} e^{\frac{-t y^{2}}{2 \pi \alpha^{\prime}}} \frac{\theta_{11}\left(\frac{i \phi t}{2 \pi}, i t\right)^{4}}{\eta(i t)^{9} \theta_{11}\left(\frac{i \phi t}{\pi}, i t\right)} .
$$

The NS ground state energy of the system is $E_{N S}^{0}=-\frac{1}{2}+\frac{\phi}{2 \pi}$ but this is projected out by GSO. The first excited state (which survives the projection) is $\psi_{-\frac{1}{2}+\frac{\phi}{\pi}} \mid 0>_{N S}$ and has mass given by

$$
m^{2}=\frac{y^{2}}{4 \pi^{2} \alpha^{\prime 2}}-\frac{\phi}{2 \pi \alpha^{\prime}}
$$

This becomes tachyonic at distances less than a critical distance $y_{c} \equiv 2 \pi \alpha^{\prime} \phi$. If we look at the large $t$ asymptotics of $(2.2)$ we find

$$
V \simeq-L^{3} \int_{0}^{\infty} \frac{d t}{t}\left(8 \pi^{2} \alpha^{\prime} t\right)^{-2} e^{-\frac{t y^{2}}{2 \pi \alpha^{\prime}}+\phi t}
$$


which diverges at $y^{2}<2 \pi \alpha^{\prime} \phi$ in agreement with (2.4).

We note that $\phi=\pi$ is equivalent to replacing one of the branes by an anti-brane 2 . In this case the tachyonic mode reflects the annihilation of the brane anti-brane pair into the vacuum.

The tachyonic mode represents an instability in the system for distances less than $y_{c}$. In particular at zero distance one expects the state to decay into the BPS state corresponding to the vector sum of the two RR charges of the branesw. If the two states have $R R$ charges $\mathbf{Q}_{1,2}$ with unit magnitude then $\mathbf{Q}_{1} \cdot \mathbf{Q}_{2}=\cos \phi$, and the mass of this state is given byt,

$$
M_{p}(\phi)=L^{p} T_{p}\left|\mathbf{Q}_{1}+\mathbf{Q}_{2}\right|=\sqrt{2} L^{p} T_{p}(1+\cos \phi)^{\frac{1}{2}}=2 L^{p} T_{p} \cos \frac{\phi}{2},
$$

where $T_{p}$ is the p-brane tension and $L(\rightarrow \infty)$ is the size of the spatial directions in which the brane is wound.

Note that the above result makes sense only in the compactified case where (at least) the plane of rotation is on a two torus. The factor $2 \cos \frac{\phi}{2}$ is just the geometrical factor that comes from the length of the final brane if we had started with initial branes of unit length.

It is possible that our conjecture that the two rotated brane configuration decays into the above BPS state can be established along the lines of [11] and [3]. The case considered in [11] is one where tachyon condensation on the D0-D2 system (or a Dp-D(p2) system) gives a $D(p-2)$ brane with magnetic flux on it. Our case is related to this in that a rotated brane is T-dual to one with magnetic flux on it. Thus we believe that the conjecture is very plausible since the above BPS state is the lowest state with the same quantum numbers as the starting configuration. One puzzling aspect of this however is that the tachyon would be expected to be localized to the region of intersection of the two branes, but its condensation must result in changing the energy density over the whole configuration. This issue of course obtains even in tachyon condensation in, for instance, the D2-D0 case, where the tachyon would be localized at the location of the D0-brane. This is a question that needs to be investigated further .

\section{Boundary states}

The boundary state formalism was used to discuss open closed string duality in a series of papers by the authors of [12] and in 13 . Here we will construct boundary states

\footnotetext{
${ }^{2}$ This is because at $\phi=\pi$ the RR exchange term flips sign compared to its value at $\phi=0$

${ }^{3}$ It has been argued in 10 that in the case when all directions are non-compact, the D-branes reconnect and move apart indefinitely. On the other hand in the compact case there is a lower bound to the energy and the scenario discussed in this paper should be valid when we consider the system with the plane in which the rotation is made to be a two torus with radii tending to infinity.

${ }^{4}$ This can be established by an argument along the lines of the one given on page 168 of [10].

${ }^{5}$ I wish to thank A. Sen for commenting on this.

${ }^{6}$ This formalism was extended to the discussion of D-branes in 14. For references on the boundary state formalism which are closely related to the discussion in this section see [15, [16, [17].
} 
corresponding to branes at angles and check open closed string duality. Instead of working with rotated branes we will turn on a ten dimensional gauge field (with constant field strength $F$ ) and follow closely the discussion in [12]. In the D-brane context the field strength components in the parallel parallel directions will be magnetic fields living on the D-brane while the parallel perpendicular field strengths will correspond to a rotation of the brane through the relation $X^{\perp}=2 \pi \alpha^{\prime} F_{\|}^{\perp} X^{\|}$. Henceforth we will write $2 \pi \alpha^{\prime} F \rightarrow F$.

The NSNS boundary state in the presence of $F$ is given by

$\left|F, \eta, k>_{N S N S}=N(F) \operatorname{det}(1+F)^{\frac{1}{2}} \exp \left\{\sum_{n=1}^{\infty} \frac{1}{n} \alpha_{-n}^{\mu} T(F)_{\mu \nu} \tilde{\alpha}_{-n}^{\nu}+i \eta \sum_{r=\mathcal{Z}^{+}-\frac{1}{2}} \psi_{-r}^{\mu} T_{\mu \nu} \tilde{\psi}_{-r}^{\nu}\right\}\right| \eta, k>_{N S N S}^{0}$

and the RR sector one by,

$$
\left|F, \eta, k>_{R R}=4 i N(F) \exp \left\{\sum_{n=1}^{\infty} \frac{1}{n} \alpha_{-n}^{\mu} T(F)_{\mu \nu} \tilde{\alpha}_{-n}^{\nu}+i \eta \sum_{r=\mathcal{Z}^{+}} \psi_{-r}^{\mu} T_{\mu \nu} \tilde{\psi}_{-r}^{\nu}\right\}\right| F, \eta, k>_{R R}^{0},
$$

where

$$
\left|F, \eta, k>_{R R}^{0}=\operatorname{tr} \exp \left(-\frac{1}{2} \psi_{\mp}^{\nu} F_{\mu \nu} \psi_{ \pm}^{\mu}\right)\right| \eta, k>_{R R}^{0}
$$

and $T(F)=\frac{1-F}{1+F} T_{0}$ with $T_{0}$ a diagonal matrix with -1 in the $\mathrm{N}$ directions and +1 in the D directions. $\eta= \pm$ and $\psi_{ \pm}=\frac{1}{\sqrt{2}}\left(\psi_{0} \pm i \tilde{\psi}_{0}\right)$ with $\psi_{\eta}^{\|}\left|\eta, k>_{R R}^{0}=\psi_{-\eta}^{\perp}\right| \eta, k>_{R R}^{0}=0$. Note that the Born-Infeld pre-factor in the NSNS state is derived in 12 and the relative normalization of the NSNS and the RR states is a consequence of supersymmetry as shown there. The above are obtained from those given in [12] by T-duality. The absolute normalization will be fixed later by comparison with the open string calculation [10] reviewed in the previous section. The GSO projected boundary states are then

$$
\mid U, F>_{N S N S}=\frac{1}{\sqrt{2}}\left(\left|F .+>_{N S N S}+\right| F .->_{N S N S}\right),
$$

and

$$
\mid U, F>_{R R}=\frac{1}{\sqrt{2}}\left(\left|F .+>_{R R}+\right| F .->_{R R}\right) .
$$

The D-p-brane state is then given by

$$
\mid D, F>=\frac{1}{\sqrt{2}}\left(\left|U, F>_{N S N S}+\right| U, F>_{R R}\right) .
$$

Choosing a basis where the $F$ field is skew diagonal we may put

$$
F_{\mu \nu}=\oplus\left(\begin{array}{cc}
0 & f_{i} \\
-f_{i} & 0
\end{array}\right), f_{i}=\tan \phi_{i}
$$

We take as in the previous section the coordinates $\mathbf{x}_{\|}=\left(x^{2}, x^{4}, x^{6}, x^{8}\right)$ as the directions along one of the branes and $\mathbf{x}_{\perp}=\left(x^{3}, x^{5}, x^{7}, x^{9}\right)$ as the $\mathrm{D}$ directions (with $x^{0}, x^{1}$ as light cone directions). 
Defining the projection matrices $P_{ \pm}(F)=\frac{1}{2}(1 \pm T(F))$, the momentum eigenstate may be written as $|k>=| P_{+}(F) k_{R}>$ in terms of the right moving momentum eigenvalue $k_{R}$. The states are normalized as $\langle k| k^{\prime}>=\delta\left(k^{\prime}-k\right)$, and the transverse position state is then given by,

$$
\left|x>=\int d k \delta\left(P_{-}(F) k\right) e^{-i\left(P_{+}(F) k\right)^{T} \cdot x}\right| k>
$$

In evaluating the matrix element of $e^{-s H_{c}}$ between D-brane states we therefore get integrals of the form, (say in the 2-3 plane)

$$
\int d k_{2}^{\prime} d k_{3}^{\prime} \int d k_{2} d k_{3} \delta\left(k_{2}^{\prime}\right) \delta\left[\frac{1}{2}(1+\cos 2 \phi) k_{2}+\frac{1}{2} \sin 2 \phi k_{3}\right] e^{-i x_{3} k_{3}^{\prime}-\frac{1}{2} \alpha^{\prime} s k_{3}^{\prime 2}} \delta^{2}\left(k-k^{\prime}\right)=\frac{2}{\sin 2 \phi}
$$

When $\phi=0, \pi$, we have putting $\delta\left(k^{\prime}-k\right)=\frac{L}{2 \pi} \delta\left(k_{\perp}^{\prime}-k_{\perp}\right)$ in the integral a factor,

$$
\frac{L}{2 \pi} \sqrt{\frac{2 \pi}{\alpha^{\prime} s}} e^{-\frac{x_{\perp}^{2}}{2 \alpha^{\prime} s}} .
$$

There is a similar factor associated with the light cone direction.

Putting all this together we have the following results. Define

$$
\begin{aligned}
\int_{s} & \equiv N(0) N(F) \prod_{i=1}^{4} \frac{2}{\sin \phi_{i}} 2^{4} \int_{0}^{\infty} d s \sqrt{\frac{2 \pi}{\alpha^{\prime} s}} e^{\frac{-y^{2}}{2 \alpha^{\prime} s}} \\
\int_{t} & \equiv N(0) N(F) \prod_{i=1}^{4} \frac{2}{\sin \phi_{i}} 2^{4} \int_{0}^{\infty} \frac{\pi d t}{t} \sqrt{\frac{2}{\alpha^{\prime} t}} e^{\frac{-y^{2} t}{2 \alpha^{\prime} \pi}} .
\end{aligned}
$$

where $y$ is, as in the previous section, the distance between the branes. Then we have

$$
\begin{gathered}
\int_{0}^{\infty} d s<0, \pm\left|e^{-s H_{c}}\right| F, \pm>_{N S N S}=\int_{s} \prod_{i=1}^{4} \tan \phi_{i} \frac{\theta_{00}\left(\frac{\phi_{i}}{\pi}, \frac{i s}{\pi}\right)}{\theta_{11}\left(\frac{\phi_{i}}{\pi}, \frac{i s}{\pi}\right)} \\
=\int_{t} \prod_{i=1}^{4} \tan \phi_{i} \frac{\theta_{00}\left(\frac{-i t \phi_{i}}{\pi}, i t\right)}{\theta_{11}\left(\frac{-i t \phi_{i}}{\pi}, i t\right)}=\int_{0}^{\infty} \frac{d t}{2 t} \operatorname{tr}_{N S} e^{-2 \pi t H_{o}} \\
\int_{0}^{\infty} d s<0, \pm\left|e^{-s H_{c}}\right| F, \mp>_{N S N S}=\int_{s} \prod_{i=1}^{4} \tan \phi_{i} \frac{\theta_{01}\left(\frac{\phi_{i}}{\pi}, \frac{i s}{\pi}\right)}{\theta_{11}\left(\frac{\phi_{i}}{\pi}, \frac{i s}{\pi}\right)} \\
=\int_{t} \prod_{i=1}^{4} \tan \phi_{i} \frac{\theta_{10}\left(\frac{-i t \phi_{i}}{\pi}, i t\right)}{\theta_{11}\left(\frac{-i t \phi_{i}}{\pi}, i t\right)}=\int_{0}^{\infty} \frac{d t}{2 t} \operatorname{tr}_{R} e^{-2 \pi t H_{o}} . \\
\int_{0}^{\infty} d s<0, \pm \mid e^{-s H_{c} \mid F, \pm>_{R R}}=-\int_{s} \prod_{i=1}^{4} \tan \phi_{i} \frac{\theta_{10}\left(\frac{\phi_{i}}{\pi}, \frac{i s}{\pi}\right)}{\theta_{11}\left(\frac{\phi_{i}}{\pi}, \frac{i s}{\pi}\right)} \\
=-\int_{t} \int_{t} \prod_{i=1}^{4} \tan \phi_{i} \frac{\theta_{01}\left(\frac{-i t \phi_{i}}{\pi}, i t\right)}{\theta_{11}\left(\frac{-i t \phi_{i}}{\pi}, i t\right)}=\int_{0}^{\infty} \frac{d t}{2 t} \operatorname{tr}_{N S}(-1)^{F} e^{-2 \pi t H_{o}} .
\end{gathered}
$$




$$
\begin{aligned}
& \int_{0}^{\infty} d s<0, \pm\left|e^{-s H_{c}}\right| F, \mp>_{R R}=-\int_{s} \prod_{i=1}^{4} \tan \phi_{i} \frac{\theta_{11}\left(\frac{\phi_{i}}{\pi}, \frac{i s}{\pi}\right)}{\theta_{11}\left(\frac{\phi_{i}}{\pi}, \frac{i s}{\pi}\right)} \\
&=-\int_{t} \prod_{i=1}^{4} \tan \phi_{i} \frac{\theta_{11}\left(\frac{-i t \phi_{i}}{\pi}, i t\right)}{\theta_{11}\left(\frac{-i t \phi_{i}}{\pi}, i t\right)}=-\int_{0}^{\infty} \frac{d t}{t} \operatorname{tr}_{R}\left(-1^{F}\right) e^{-2 \pi t H_{o}} .
\end{aligned}
$$

The second equality in each of the above equations is the result of the transformation $s \leftarrow t=\frac{\pi}{s}$. The subscripts on the trace refers to the states (NS or R) over which the trace is taken. The last equality follows from comparison with the calculation of the previous section (the open string loop calculation) and is valid if we choose the normalization constant to be $N(F)=\frac{1}{8 \sqrt{2} \pi} \prod_{i} \cos ^{2} \phi_{i}$. With this we have (using (3.6) and (3.5))the result

$$
\int_{0}^{\infty} d s<D, 0\left|e^{-s H_{c}}\right| D, F>=\int_{0}^{\infty} \frac{d t}{t} \operatorname{tr}_{N S-R} \frac{1}{2}\left(1+(-1)^{F}\right) e^{-2 \pi t H_{o}} .
$$

This checks the open string closed string duality for rotated branes. In particular when three of the phis are zero and one is equal to $\pi$ we get the result for brane anti-brane interaction given in section 2.1 of [2].

\section{Rotated D-branes on a $Z_{2}$ orbifold}

Let us, following Sen [2], consider an orbifold (of IIB string theory) generated by

$$
g=(-1)^{F_{L}} I_{4}, \quad I_{4}: x^{6,7,8,9} \rightarrow-x^{6,7,8,9}
$$

where $(-1)_{L}^{F}$ acts as -1 on the left moving Ramond ground state and is +1 on all other ground states. For concreteness we again consider a 3 -brane but this time we take

$$
\mathbf{x}_{\|}=\left\{x^{2}, x^{3}, x^{4}, x^{8}\right\}, \quad \mathbf{x}_{\perp}=\left\{x^{5}, x^{6}, x^{7}, x^{9}\right\} .
$$

The light cone directions are as before $\left\{x^{0}, x^{1}\right\}$ and we are working again in the double Wick rotated formalism where $x^{2}$ is the Euclideanized time. We take one brane to be stretched along the above $x_{\|}$directions and the other to be parallel to it in the 2,3 and 8 directions but rotated with respect to it in the $4-5$ plane by an angle $\phi$. As in [2] one can check that

$$
g\left|U, F>_{N S N S}=\right| U, F>_{N S N S}, \quad g\left|U, F>_{R R}=\right| U, F>_{R R} .
$$

In addition to these untwisted states there are twisted states that can be represented as

$$
\begin{aligned}
\mid F, \eta, k>_{N S N S ; T}= & N(F) \operatorname{det}(1+F)^{\frac{1}{2}} \\
& \exp \left\{\sum_{n} \frac{1}{n} \alpha_{-n}^{\mu} T(F)_{\mu \nu} \tilde{\alpha}_{-n}^{\nu}+i \eta \sum_{r} \psi_{-r}^{\mu} T_{\mu \nu}(F) \tilde{\psi}_{-r}^{\nu}\right\} \mid \eta, k>_{N S N S ; T}^{0},
\end{aligned}
$$




$$
\left|F, \eta, k>_{R R ; T}=4 i N(F) \exp \left\{\sum_{n} \frac{1}{n} \alpha_{-n}^{\mu} T(F)_{\mu \nu} \tilde{\alpha}_{-n}^{\nu}+i \eta \sum_{r} \psi_{-r}^{\mu} T_{\mu \nu} \tilde{\psi}_{-r}^{\nu}\right\}\right| F, \eta, k>_{R R ; T}^{0} .
$$

In the above sums we take

$$
\begin{array}{rrr}
N S N S: \quad n \quad & \in \quad \mathcal{Z}_{+} ; \mu=2,3,4,5 ; \quad r \in \mathcal{Z}_{+}-\frac{1}{2} \\
& \epsilon \quad \mathcal{Z}_{+}-\frac{1}{2} ; \mu=6,7,8,9 ; \quad r \epsilon \mathcal{Z}_{+}, \\
R R: \quad n, r \quad & \in \quad \mathcal{Z}_{+} ; \mu=2,3,4,5 ; \\
& \in \quad \mathcal{Z}_{+}-\frac{1}{2} ; \mu=6,7,8,9 .
\end{array}
$$

Because of the orbifold condition the momentum integration is just over the perpendicular directions that are not transverse to the orbifold fixed plane. So we define

$$
\begin{aligned}
\mid \eta>_{N S N S ; T} & =2 \tilde{N} \int d k^{0} d k^{1} \mid k, \eta> \\
\mid \eta>_{R R ; T} & =2 \tilde{N} \int d k^{0} d k^{1} \mid k, \eta>.
\end{aligned}
$$

As in [2] the GSO invariant twisted states are then given by

$$
\begin{aligned}
\mid T>_{N S N S} & \left.=\frac{1}{\sqrt{2}}\left(\mid+>_{N S N S ; T}\right)+\mid->_{N S N S ; T}\right) \\
\mid T>_{R R} & \left.=\frac{1}{\sqrt{2}}\left(\mid+>_{R R ; T}\right)+\mid->_{R R ; T}\right) .
\end{aligned}
$$

The untwisted matrix elements are given by the same formulae as before (3.12-3.15) with the replacements appropriate to the case when three of the angles are zero (see remark after equation (2.1)). For the twisted ones we now get,

$$
\begin{aligned}
\int_{0}^{\infty} d s<0, \pm\left|e^{-s H_{c}}\right| F, \pm>_{N S N S ; T} & =\tilde{N}(0) \tilde{N}(F) \frac{2 \pi}{\alpha^{\prime}} \int \frac{d t}{t} \frac{\sin \phi}{\cos \phi} \frac{\theta_{00}\left(\frac{-i t \phi}{\pi}, i t\right) \theta_{00}(0, i t) \theta_{01}(0, i t)^{2}}{\eta(i t)^{3} \theta_{11}\left(\frac{-i t \phi}{\pi}, i t\right) \theta_{10}(0, i t)^{2}} \\
& =\int_{0}^{\infty} \frac{d t}{2 t} \operatorname{tr}_{N S, \phi} e^{-2 \pi t H_{0}} g \\
\int_{0}^{\infty} d s<0, \mp\left|e^{-s H_{c}}\right| F, \pm>_{N S N S ; T} & =0=-\int_{0}^{\infty} \frac{d t}{2 t} \operatorname{tr}_{R, \phi} e^{-2 \pi t H_{0}} g \\
\int_{0}^{\infty} d s<0, \pm\left|e^{-s H_{c}}\right| F, \pm>_{R R ; T} & =\tilde{N}(0) \tilde{N}(F) \frac{2 \pi}{\alpha^{\prime}} \int \frac{d t}{t} \frac{\sin \phi}{\cos \phi} \frac{\theta_{01}\left(\frac{-i t \phi}{\pi}, i t\right) \theta_{01}(0, i t) \theta_{00}(0, i t)^{2}}{\eta(i t)^{3} \theta_{11}\left(\frac{-i t \phi}{\pi}, i t\right) \theta_{10}(0, i t)^{2}} \\
& =\int_{0}^{\infty} \frac{d t}{2 t} \operatorname{tr}_{N S, \phi} e^{-2 \pi t H_{0}}(-1)^{F} \cdot g, \\
\int_{0}^{\infty} d s<0, \mp\left|e^{-s H_{c}}\right| F, \pm>_{R R ; T} & =0=-\int_{0}^{\infty} \frac{d t}{2 t} \operatorname{tr}_{R, \phi} e^{-2 \pi t H_{0}}(-1)^{F} \cdot g
\end{aligned}
$$


We have performed the transformation $s=\frac{\pi}{t}$ in the expressions above and then compared with the corresponding open string calculation. As in the previous discussion these equations will also thus fix the overall normalization constant $\tilde{N}(F)$.

The complete D-brane state is then defined (after changing the notation slightly by replacing $F$ in the state by the angle of rotation $\phi$ )

$$
\mid D ; \phi, \pm>=\frac{1}{2}\left[\left|U, \phi>_{N S N S}+\right| U, \phi>_{R R} \pm\left(\left|T, \phi>_{N S N S}+\right| T, \phi>_{R R}\right)\right.
$$

where the states on the right hand side are defined in (3.5) and (4.9). It should be noted that the anti-brane state is given as $|\bar{D} ; 0, \pm>=| D ; \pi, \pm>$. Using the above results we then have

$$
\begin{aligned}
\int_{0}^{\infty} d s<D ; 0 \pm\left|e^{-s H_{c}}\right| D ; \phi, \pm> & =\int_{0}^{\infty} \frac{d t}{2 t} \operatorname{tr}_{N S-R, \phi} e^{-2 \pi t H_{0}} \frac{1}{2}\left(1+(-1)^{F}\right) \cdot \frac{1}{2}(1+g) . \\
\int_{0}^{\infty} d s<D ; 0 \pm\left|e^{-s H_{c}}\right| D ; \phi, \mp> & =\int_{0}^{\infty} \frac{d t}{2 t} \operatorname{tr}_{N S-R, \phi} e^{-2 \pi t H_{0}} \frac{1}{2}\left(1+(-1)^{F}\right) \cdot \frac{1}{2}(1-g) .
\end{aligned}
$$

Note that the effect of replacing one of the branes by an anti-brane i.e. $\phi=\pi$ on the right hand side of this is equivalent to putting $\phi=0$ but changing the sign in front of $(-1)^{F}$.

Now still following Sen [2] we compactify the eight direction on a circle of radius $\mathrm{R}$ and put a Wilson line on one of the D-branes. The wave function then acquires a phase $e^{i \chi}$ (say) as one goes round the circle i.e. $x^{8} \rightarrow x^{8}+2 \pi R$, and $g$ invariance restricts the values of $\chi$ to $0, \pi$. Now in the compactified space the orbifold action gives two fixed planes at $x^{8}=0, \pi R$. So it is convenient to start with a circle of radius $2 R$ and introduce two groups [2],

$$
\begin{array}{ll}
g_{1}: & x^{6} \rightarrow-x^{6}, \quad x^{7} \rightarrow-x^{7}, \quad x^{8} \rightarrow-x^{8} x^{9} \rightarrow-x^{9}, \quad \text { and }(-1)^{F_{L}} \\
g_{2}: & x^{6} \rightarrow-x^{6}, \quad x^{7} \rightarrow-x^{7}, \quad x^{8} \rightarrow-x^{8}+2 \pi R x^{9} \rightarrow-x^{9}, \quad \text { and }(-1)^{F_{L}} .
\end{array}
$$

Then we also have

$$
g_{2} g_{1}: x^{8} \rightarrow x^{8}+2 \pi R
$$

so that modding out by this symmetry gives us back a circle of radius $R$. Now we may construct boundary states for this configuration by adding to the untwisted states the two twisted sectors at the two orbifold planes.

$$
\begin{aligned}
\mid D ; \chi, \phi, \epsilon> & =\frac{1}{2}\left[\left|U ; \chi, \phi>_{N S N S}+\right| U ; \chi, \phi>_{R R}+\epsilon \frac{1}{\sqrt{2}}\left(\left|T_{1}, \phi>_{N S N S}+\right| T_{1}, \phi>_{R R}\right)\right. \\
& \left.+e^{i \chi} \epsilon \frac{1}{\sqrt{2}}\left(\left|T_{2}, \phi>_{N S N S}+\right| T_{2}, \phi>_{R R}\right)\right]
\end{aligned}
$$

with $\epsilon= \pm$. To compare with the corresponding boundary state that Sen constructed [2] we note that for $\phi=0, \pi,\left|U ; \chi, \phi>_{R R}=\epsilon_{1}(\phi)\right| U ; \chi, 0>_{R R}$ and $\mid T_{1,2}, \phi>_{N S N S}=$ 
$\left|T_{1,2}, 0>_{N S N S},\right| T_{1}, \pi>_{R R}=\epsilon_{1} \mid T_{1}, 0>_{R R}$ where $\epsilon_{1,2}(\phi)= \pm 1$ for $\phi=0, \pi$ respectively and $\epsilon=\epsilon_{2}$.

Now we may compute the interaction between two such states as before to get,

$$
\int_{0}^{\infty} d s<D ; 0,0,0\left|e^{-s H_{c}}\right| D ; \chi, \phi, \epsilon>=\int \frac{d t}{2 t} \operatorname{tr}_{N S-R, \phi} e^{-2 \pi t H_{0}} \frac{1}{8}\left(1+(-1)^{F}\right) \cdot\left(1+\epsilon g_{1}\right) \cdot\left(1+e^{i \chi} \epsilon g_{2}\right) .
$$

\section{Tachyon condensation}

Let us label the D-branes by the coefficients $q_{1}=\epsilon, q_{2}=\epsilon e^{i \chi}$ of the twisted sector states $\mid T 1,2>$ at each orbifold plane. There are four possibilities for $\left(q_{1}, q_{2}\right)$; a) $\left.(+,+), \mathrm{b}\right)(-,-)$, c) $(+,-), \mathrm{d})(-,+)$. If we take a) and c) together then the masses (per unit volume in the fixed plane directions) in the ground state, after tachyon condensation, of the states at the two ends $0, \pi R$ of the compact direction may be written as $m_{++}$at one end and $m_{+-}$ at the other. This is a situation with a Wilson line $(\chi=\pi)$ at one end. The tachyon field is then anti-periodic in the eighth direction and the corresponding momentum is quantized in half integral units $k_{8}=\frac{n+\frac{1}{2}}{R}$. The lowest mode then has mass

$$
m^{2}(\phi)=\frac{1}{4 R^{2}}-\frac{\phi}{2 \pi \alpha^{\prime}}
$$

This becomes marginal at the critical radius

$$
R_{c}=\sqrt{\frac{\pi \alpha^{\prime}}{2 \phi}}
$$

As in [2] at this radius the mass (per unit volume in the fixed plane directions) of the configuration may be taken to be that of the two branes (taken now to be p-branes),

$$
M_{c}(\phi)=2 \pi T_{p} R_{c}=2 \pi \frac{1}{g(2 \pi)^{p}\left(\sqrt{\alpha}^{\prime}\right)^{p+1}} \sqrt{\frac{\pi \alpha^{\prime}}{2 \phi}}=\frac{1}{g(2 \pi)^{(p-1)}\left(\sqrt{\alpha}^{\prime}\right)^{p}} \sqrt{\frac{\pi}{2 \phi}}=\sqrt{\frac{\pi}{2 \phi}} T_{p-1} .
$$

The above depends on the assumption that at the critical radius $R_{c}$ (above which a tachyonic mode appears) the original configuration becomes degenerate with the final one. This is tantamount to the assumption that the tachyon is exactly marginal at the critical radius. Unfortunately unlike in the brane anti-brane case where there is a conformal field theory proof [4], for general angles we are unable to do better than the above heuristic argument.

Now in section 2 we argued that the unstable configuration of two non-parallel D branes in flat space decays into a BPS state with mass given by (2.5). Let us write $m_{s}=M_{p}(\phi) \pi R / L^{p}$. In the present case the tachyon has kinks at the orbifold fixed planes $x^{8}=0, \pi R$ (see [2]) and correspondingly we expect solitonic states localized on the fixed planes. Then for radius $\mathrm{R}$ the mass per unit ( $\mathrm{p}-1)$ volume (transverse to the compactified direction) of the ground state configuration is conjectured to be given by, 


$$
\begin{aligned}
m_{++}+m_{+-}+m_{s} & =2 \pi R_{c} T_{p-1}+\pi\left(R-R_{c}\right) T_{p} \sqrt{2}(1+\cos \phi)^{\frac{1}{2}} \\
& =T_{p-1} \sqrt{\frac{\pi}{2 \phi}}\left(1-\cos \frac{\phi}{2}\right)+\pi R T_{p} \sqrt{2}(1+\cos \phi)^{\frac{1}{2}}
\end{aligned}
$$

This expression is constructed so that it gives the formula obtained above (5.3) at the critical radius $R_{c}$. At $\phi=0$ it reduces to twice the mass of the two p-branes and at $\phi=\pi$, the brane anti-brane case, one gets $T_{p-1} / \sqrt{2}$ the mass derived by Sen [2], [4]. The second term is the mass of the BPS state stretched between the two orbifold planes and so we expect the first term to be the total mass of the states localized on the orbifold planes. i.e.

$$
m_{++}+m_{+-}=T_{p-1} \sqrt{\frac{\pi}{2 \phi}}\left(1-\cos \frac{\phi}{2}\right)
$$

Now let us consider the combination of the branes a) and b). In this case there is no Wilson line but only states odd under $g_{1}$ and $g_{2}$ are projected in. The $k_{8}=0$ mode is projected out and the lowest open string mode has $k_{8}= \pm \frac{1}{R}$ and its mass is given by,

$$
m^{2}=k_{8}^{2}-\frac{\phi}{2 \pi \alpha^{\prime}}
$$

giving a critical radius of

$$
R_{c}^{\prime}=\sqrt{\frac{2 \pi \alpha^{\prime}}{\phi}}=2 R_{c} .
$$

In this situation we have a state of mass $m_{+-}$at each end. Reasoning as before this leads to the equation

$$
m_{+-}=T_{p-1} \sqrt{\frac{\pi}{2 \phi}}\left(1-\cos \frac{\phi}{2}\right)
$$

Using (5.5) this gives

$$
m_{++}=0
$$

Note that for $\phi=0, m_{+-}=0$ and for $\phi=\pi$ (the brane-anti-brane case) we get the value obtained by Sen [2].

\section{Comments}

The twisted state RR charge of the final configuration must be equal to the twisted sector charge of the initial configuration at each end. The final brane stretched between the two orbifold planes also has twisted sector charge at each end and if this does not match the initial configuration charges at either end then there must be a solitonic state at that end. Now at the $x^{8}=0$ end one may reason as follows. The twisted sector charge vector at this end is proportional to the untwisted sector charge, and given the untwisted charges $\mathbf{Q}_{\mathbf{1}}, \mathbf{Q}_{\mathbf{2}}$ of the two initial branes the total twisted sector charge of the initial 
configuration would be proportional to $\mathbf{Q}_{1}+\mathrm{Q}_{\mathbf{2}}$ (see equation (4.15) and the discussion at the begining of section 5). But the untwisted sector charge of the final brane stretched between the two fixed planes is $\mathbf{Q}_{\mathbf{1}}+\mathbf{Q}_{\mathbf{2}}$ and hence its twisted sector charge at the $x^{8}=0$ end is equal to that of the initial configuration. Hence there is no residual twisted sector charge at this end and one would expect the mass of a ++ state at this end to be zero. This is consistent with the above result $m_{++}=0$.

At the $x^{8}=\pi R$ end however the the total twisted sector $\mathrm{RR}$ charge of the initial branes is proportional to $\mathbf{Q}_{\mathbf{1}}-\mathbf{Q}_{\mathbf{2}}$ (we have taken the Wilson line $e^{i \chi}=1$ for the first brane and -1 for the second). But the untwisted sector charge of the final brane is the sum of the two charges and hence its twisted sector charge is proportional to $\pm\left(\mathbf{Q}_{\mathbf{1}}+\mathbf{Q}_{\mathbf{2}}\right)$ (here we have also used the sign ambiguity coming from the possibility of assigning either $\epsilon= \pm$ to the end of the final brane). Thus (for either sign) there is a discrepancy in the twisted sector charges at this end which must therefore indicates the presence of a $p-1$ dimensional soliton living at this end carrying charge proportional to $2 \mathbf{Q}_{\mathbf{1}}$ or $2 \mathbf{Q}_{\mathbf{2}}$. This accounts for the state with non-zero mass $m_{+-}$obtained above. The fact that this charge is independent of the angle $\phi$ while the mass $m_{+-}$depends on the angle presumably means that this $p-1$ dimensional soliton is bound to the $p$ dimensional brane and the angular dependence reflects the binding energy

\section{Acknowledgments}

I wish to thank J. Polchinski and A. Sen for helpful correspondence and the latter also for comments on the manuscript. This work is partially supported by the Department of Energy contract No. DE-FG02-91-ER-40672.

\section{References}

[1] A. Sen, JHEP 9806 (1998) 007, hep-th/9803194.

[2] A. Sen, JHEP 9808 (1998) 010, hep-th/9805019.

[3] A. Sen, JHEP 9808 (1998)012, hep-th/9805170.

[4] A. Sen, JHEP 9809 (1998) 023, hep-th/9808141.

[5] O. Bergman and M. Gaberdiel, Phys.Lett. B441 (1998) 133-140, hep-th/9806155.

[6] E. Witten, JHEP 9812 (1998) 019, hep-th/9810188; P. Horava, "Type IIA D-Branes, K-Theory, and Matrix Theory", to appear in ATMP, hep-th/9812135; S. Gukov, "KTheory, Reality, and Orientifolds hep-th/9901042'; H. Garcia-Compean, "D-branes in Orbifold Singularities and Equivariant K-Theory" hep-th/9812226; O. Bergman, E. G. Gimon, P. Horava "Brane Transfer Operations and T-Duality of Non-BPS

\footnotetext{
${ }^{7}$ This section is largely the result of comments made by an annonymous referee
} 
States", hep-th/9902160; K. Olsen, R. J. Szabo, "Brane Descent Relations in Ktheory", hep-th/9904153.

[7] M. Frau, L. Gallot, A. Lerda, P. Strigazzi, "Stable non-BPS D-branes in Type I string theory", hep-th/9903123.

[8] A. Sen, "Non-BPS States and Branes in String Theory", hep-th/9904207; A. Lerda, R. Russo, "Stable non-BPS states in string theory: a pedagogical review", hepth/9905006.

[9] O. Bergman and M. Gaberdiel, Nucl.Phys. B499 (1997) 183, hep-th/9701137.

[10] J. Polchinski, "String theory" Vol II, Cambridge (1998).

[11] E. Gava, K.S. Narain, M.H. Sarmadi, Nucl.Phys. B504 (1997) 214, hep-th/9704006.

[12] C. Callan, C Lovelace, C. Nappi and S. Yost, Nucl. Phys. B308 (1988) 221.

[13] J. Polchinski and Y. Cai, Nucl. Phys. B286 (1988) 91.

[14] M. Li, Nucl.Phys. B460 (1996) 351, hep-th/9510161; C. Schmidhuber Nucl.Phys. B467 (1996) 146, hep-th/9601003; S.P. de Alwis, K. Sato Phys.Rev. D53 (1996) 7187, hep-th/9601167.

[15] M. Green and M. Gutperle, Nucl.Phys. B476 (1996) 484, hep-th/9604091; F. Hussain, R. Iengo, C. Nez, C. Scrucca, Phys.Lett. B409 (1997) 101, hep-th/9706186; M. Bertolini, R. Iengo, C. Scrucca, Nucl.Phys. B522 (1998) 193, hep-th/9801110.

[16] P. Di Vecchia, M. Frau, I. Pesando, S. Sciuto, A. Lerda, R. Russo, Nucl.Phys. B507 (1997) 259, hep-th/9707068; M. Billo', P. Di Vecchia, M. Frau, A. Lerda, I. Pesando, R. Russo, S. Sciuto, Nucl.Phys. B526 (1998) 199, hep-th/9802088.

[17] A. Rechknagel and V. Schomerus, "Boundary Deformation Theory and Moduli Spaces of D-Branes" hep-th/9811237. 